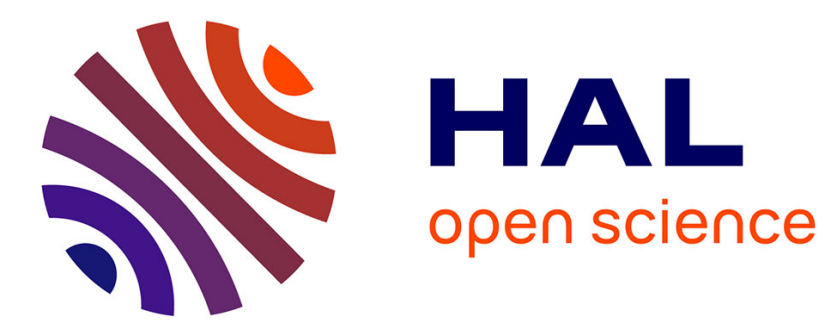

\title{
Relations entre les diverses mobilités des trous dans le silicium $\mathbf{p}$
}

J. Mercier

\section{To cite this version:}

J. Mercier. Relations entre les diverses mobilités des trous dans le silicium p. Revue de Physique Appliquée, 1969, 4 (4), pp.477-480. 10.1051/rphysap:0196900404047700 . jpa-00243314

\section{HAL Id: jpa-00243314 https://hal.science/jpa-00243314}

Submitted on 1 Jan 1969

HAL is a multi-disciplinary open access archive for the deposit and dissemination of scientific research documents, whether they are published or not. The documents may come from teaching and research institutions in France or abroad, or from public or private research centers.
L'archive ouverte pluridisciplinaire HAL, est destinée au dépôt et à la diffusion de documents scientifiques de niveau recherche, publiés ou non, émanant des établissements d'enseignement et de recherche français ou étrangers, des laboratoires publics ou privés. 


\title{
RELATIONS ENTRE LES DIVERSES MOBILITÉS DES TROUS DANS LE SILIGIUM $p$
}

\author{
- Par J. MERGIER, \\ Ingénieur G.N.R.S., L.E.P.M., Section Physique des Couches Minces, Cedex r66, 38-Grenoble-Gare.
}

(Reçu le 9 juin 1969.)

\begin{abstract}
Résumé. - Les résultats de calcul de mobilité des porteurs majoritaires dans les matériaux semiconducteurs, à partir de la géométrie de Corbino, sont interprétés dans le cas du silicium $\mathrm{p}$ par la contribution des deux espèces de trous. Les valeurs connues du rapport de leurs masses, ainsi que l'hypothèse d'un mécanisme de dispersion par phonons acoustiques rendent compte du facteur correctif qui relie la mobilité de magnétorésistance de Corbino à la mobilité de conductivité.
\end{abstract}

\begin{abstract}
The results of the calculation of the majority carriers mobility of semiconductors, using the Corbino disk, are explained for $\mathrm{p}$ type silicon by the existence of two kinds of holes. The known values of their masses' ratio, and the hypothesis of a scattering process by acoustical phonons give the correct value for the ratio of magnoteresistance to drift mobilities.
\end{abstract}

Introduction. - C'est le mérite du Laboratoire de la G.S.F. [1] d'avoir remis à l'honneur la géométrie de Corbino pour la mesure de la mobilité des porteurs majoritaires dans les couches minces semiconductrices.

Appliquée au cas des couches hétéroépitaxiques de silicium sur substrat d'alumine $\alpha$, cette méthode a conduit à de curieux résultats. Alors qu'il résultait de nos mesures, soit en effet Hall, soit en géométrie de Van der Pauw [2] que les mobilités étaient toujours inférieures, dans ce cas, à celles du matériau classique, la mobilité de Corbino, calculée à partir de la variation relative de résistance du disque semiconducteur sous l'effet du champ magnétique, était souvent supérieure à celle du matériau massique.

Nous avons très tôt pensé à faire intervenir les trous légers et lourds résultant de l'anisotropie des surfaces d'énergie du silicium p [3]. Encore convenait-il de vérifier que les résultats s'appliquaient aussi au matériau massique. Effectivement, [4] indique :

$$
\mu_{\text {Corbino }}=840 \text { et } 895 \mathrm{~cm}^{2} / \mathrm{V} . \mathrm{s}
$$

pour des échantillons massiques $\operatorname{Si}(111), \rho=5 \Omega \mathrm{cm}(\mathrm{p})$.

La correction calculée en [4] de 1,57 n'explique pas le facteur voisin de 2 entre la mobilité de Corbino et la mobilité de conductivité $\left(\mu_{\mathrm{c}} \simeq 450 \mathrm{~cm}^{2} / \mathrm{V} . \mathrm{s}\right)$.

Nous avons repris les calculs, par une extension des travaux de [5] au cas de conduction par deux types de trous.

Hypothèses. - 1) Existence des trous lourds et légers de masses effectives $m_{1}^{*}$ et $m_{2}^{*} ; 2$ ) Semiconducteur non dégénéré, non compensé, isotherme; 3) Existence d'un temps de relaxation unique pour les deux sortes de trous, caractéristique du mode de dispersion et relié à l'énergie $\varepsilon$ de ces trous par :

$$
\tau=\tau_{0} \cdot\left(\frac{\varepsilon}{k T}\right)^{r}
$$

$r$ pouvant prendre, selon les modèles les plus classiques, les valeurs : $-1 / 2,0,1 / 2,3 / 2$.

Résultats des calculs. - Geux-ci sont développés dans l'annexe. Ils traduisent une contribution additive des deux espèces de trous.

Nous rappellerons simplement les définitions de diverses mobilités :

1) Mobilité de conductivité $\mu_{\mathrm{c}}$. - Elle traduit l'effet du seul champ électrique sur le comportement macroscopique des porteurs de charge.

2) Mobilité de Hall $\mu_{\mathrm{H}}$. - Elle traduit la modification des lignes de courant sous l'action du champ magnétique $H$.

3) Mobilité de magnétorésistance de Corbino $\mu_{\mathrm{M}}$. - Elle traduit la variation relative de résistance du semiconducteur sous l'effet du champ magnétique.

Cas d'un seul type de porteur. - On trouve :

$$
\begin{aligned}
& \mu_{\mathrm{c}}=\frac{q}{m^{*}} \frac{\langle\tau\rangle}{\left\langle\tau^{0}\right\rangle} \\
& \mu_{\mathrm{H}}=\frac{q}{m^{*}} \frac{\left\langle\tau^{2}\right\rangle}{\langle\tau\rangle} \\
& \mu_{\mathrm{I}}^{2}=\frac{q^{2}}{m^{*}} \frac{\left\langle\tau^{3}\right\rangle}{\langle\tau\rangle}
\end{aligned}
$$

$\left\langle\tau^{n}\right\rangle$ étant une fonction définie en annexe. 
Dans le cas où $\tau$ obéit à la relation citée plus haut, [5] a calculé les fonctions :

$$
\begin{gathered}
f=\frac{\mu_{\mathrm{H}}}{\mu_{\mathrm{c}}}=\frac{\left\langle\tau^{2}\right\rangle\left\langle\tau^{0}\right\rangle}{\langle\tau\rangle^{2}}=\frac{\Gamma(2 r+5 / 2) \Gamma(5 / 2)}{\{\Gamma(r+5 / 2)\}^{2}} \\
\theta=\left(\frac{\mu_{\mathrm{M}}}{\mu_{\mathrm{H}}}\right)^{2}=\frac{\left\langle\tau^{3}\right\rangle\langle\tau\rangle}{\left\langle\tau^{2}\right\rangle^{2}}=\frac{\Gamma(3 r+5 / 2) \Gamma(r+5 / 2)}{\{\Gamma(2 r+5 / 2)\}^{2}} .
\end{gathered}
$$

Elles sont reportées sur la figure 1 en fonction de $r$.

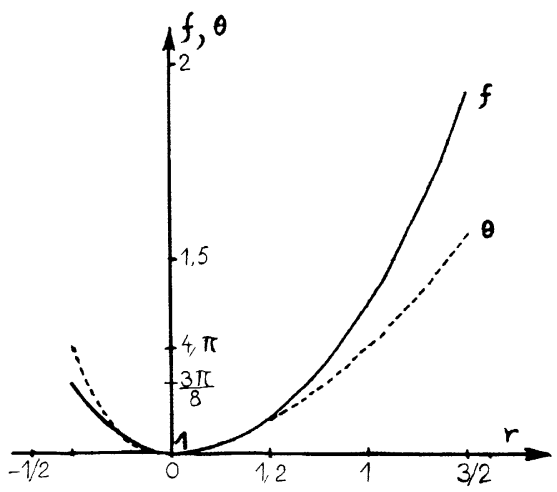

FIG. 1. - Fonctions $f(r), \theta(v)$ caractéristiques du mode de dispersion en fonction de l'indice $r$ de dispersion.

Cas de deux types de porteurs. - On introduit la masse relative des trous $m=\frac{m_{1}^{*}}{m_{2}^{*}}$, [3] donne $m=3$
et l'on trouve :

$$
\begin{gathered}
\frac{\mu_{\mathrm{M}}}{\mu_{\mathrm{c}}}=f \cdot \sqrt{ } \bar{\theta} \cdot \frac{\left(1+m^{3 / 2}\right)^{1 / 2}\left(1+m^{-3 / 2}\right)}{\left(1+m^{-1 / 2}\right)^{3 / 2}} \\
\frac{\mu_{\mathrm{H}}}{\mu_{\mathrm{c}}}=f \cdot \frac{\left(1+m^{1 / 2}\right)\left(1+m^{-3 / 2}\right)}{\left(1+m^{-1 / 2}\right)^{2}}
\end{gathered}
$$

$\mu_{\mathrm{c}}$ étant la mobilité de conductivité pour le cas d'existence des deux types de trous.

Soit avec $m=3$ :

$$
\mu_{\mathrm{c}}=1,32 \mu_{1}
$$

$\mu_{1}$ étant la mobilité des trous lourds.

Vérification. - L'application numérique donne :

$$
\begin{aligned}
& \mu_{\mathrm{c}}=1,32 \mu_{1} \\
& \mu_{\mathrm{H}}=1,32 \cdot f \cdot \mu_{\mathrm{c}} \\
& \mu_{\mathrm{M}}=1,5 \cdot f \cdot \sqrt{\theta} .
\end{aligned}
$$

Or, dans le cas de dispersion due aux phonons acoustiques, que l'on admet en général :

$$
\begin{gathered}
r=-1 / 2 ; \quad f=1,18 ; \quad \theta=1,28 \\
\frac{\mu_{\mathrm{H}}}{\mu_{\mathrm{c}}}=1,56 ; \quad \frac{\mu_{\mathrm{M}}}{\mu_{\mathrm{c}}}=2 .
\end{gathered}
$$

Conclusion. - Les calculs précédents étant vérifiés expérimentalement pour le matériau massique, il en résulte que l'on peut espérer tirer des renseignements de cette mesure, locale, dans le cas des couches hétéroépitaxiques. Néanmoins, il importe d'être prudent dans l'interprétation des résultats car il semble établi par ailleurs que :

1) De telles couches sont souvent compensées [6];

2) Les mécanismes de dispersion soient assez complexes (collisions sur des atomes neutres).

Il en résulte une certaine incertitude sur l'exposant $r$ qui rentre dans $f$ et $\theta$. Une étude en fonction de la température serait alors très utile.

Remerciements. - Je remercie vivement MM. Jund et Poirier, du Laboratoire de Recherches PhysicoGhimiques de la C.S.F., pour les fructueuses discussions sur le sujet, ainsi que M. le Professeur Rodot pour les conseils.

\section{ANNEXE}

Cas d'un seul type de porteurs. - 1) En géométrie de Corbino, le champ électrique est radial $\left(E_{1}, 0,0\right)$, le champ magnétique axial $(0,0, H)$, le courant a deux composantes $\left(J_{1}, J_{2}, 0\right)$ :

$$
\begin{gathered}
J_{1}=\frac{q \hbar}{m^{*}} \beta K_{1}(H) \cdot E_{1} \\
J_{2}=\frac{q \hbar}{m^{*}} \beta L_{1}(H) \cdot E_{1} \\
\beta=\frac{\left(2 m^{*}\right)^{3 / 2} q}{3 \pi^{2} \hbar^{4}} .
\end{gathered}
$$

Pour les champs magnétiques faibles, $\omega_{\mathrm{c}} \tau \ll 1$, avec :

$$
\omega_{\mathrm{c}}=\frac{q H}{m^{*}}-\frac{}{c}
$$

$\left(c=10^{+8}\right.$ pour $H$ gauss, $m^{*}(\mathrm{~g})$ et $q$ en $\left.(\mathrm{Cb})\right)$.

Les fonctions précédentes sont pour des champs faibles :

$$
\begin{aligned}
& K_{1}(H) \simeq\langle\tau\rangle-\omega_{\mathrm{c}}^{2}\left\langle\tau^{3}\right\rangle \\
& L_{1}(H) \simeq \omega_{\mathrm{c}}\left\langle\tau^{2}\right\rangle \\
&\left\langle\tau^{n}\right\rangle \equiv \int_{0}^{\infty} \varepsilon^{3 / 2} \tau^{n} \frac{\partial f_{0}}{\partial \varepsilon} \mathrm{d} \varepsilon \quad n \text { entier } \geqslant 0
\end{aligned}
$$

$f_{0}$ : probabilité d'occupation du niveau $\varepsilon$ en champ nul.

La mobilité de conductivité $\mu_{\mathrm{c}}$ est :

$$
\mu_{\mathrm{c}}=\frac{q}{m^{*}} \cdot \frac{\langle\tau\rangle}{\left\langle\tau^{0}\right\rangle}
$$


La mobilité de Hall $\mu_{\mathrm{H}}$ est définie par :

$$
\frac{\mu_{\mathrm{H}} \cdot H}{c}=\frac{J_{2}}{J_{1}}
$$

et négligeant les termes en $H^{2}$ :

$$
\begin{gathered}
\mu_{\mathrm{H}}=\frac{q}{m^{*}} \cdot \frac{\left\langle\tau^{2}\right\rangle}{\langle\tau\rangle} \\
f=\frac{\mu_{\mathrm{H}}}{\mu_{\mathrm{c}}}=\frac{\left\langle\tau^{2}\right\rangle\left\langle\tau^{0}\right\rangle}{\langle\tau\rangle^{2}} \text { est calculée en }[5], \\
=\frac{\Gamma(2 r+5 / 2) \Gamma(5 / 2)}{\{\Gamma(r+5 / 2)\}^{2}}
\end{gathered}
$$

$\Gamma(x)$ fonction eulérienne de $1^{\text {re }}$ espèce.

La mobilité de magnétorésistance de Corbino $\mu_{M}$ se déduit de la variation relative de résistance :

$$
\frac{\Delta \rho}{\rho_{0}}=\left(\frac{\mu_{M} H}{c}\right)^{2} \text {. }
$$

Cette définition est cohérente car $\mu_{M}$ est indépendant de $H$ dans une large gamme de champ magnétique :

d'où :

$$
\begin{gathered}
J_{1}=\sigma_{1}(H) \cdot E_{1} \\
\frac{\Delta \rho}{\rho_{0}}=\frac{\sigma_{0}-\sigma}{\sigma_{0}}=\omega_{\mathrm{c}}^{2} \frac{\left\langle\tau^{3}\right\rangle}{\langle\tau\rangle}
\end{gathered}
$$

$$
\mu_{\mathrm{M}}=\frac{q}{m^{*}}\left[\frac{\left\langle\tau^{3}\right\rangle}{\langle\tau\rangle}\right]^{1 / 2}
$$

et :

$$
\frac{\mu_{\mathrm{M}}}{\mu_{\mathrm{H}}}=\sqrt{\bar{\theta}}=\frac{\sqrt{\left\langle\tau^{3}\right\rangle\langle\tau\rangle}}{\left\langle\tau^{2}\right\rangle}
$$

calculée par [5]

$$
\theta=\frac{\Gamma(3 r+5 / 2) \Gamma(r+5 / 2)}{[\Gamma(2 r+5 / 2)]^{2}}
$$

Cas de deux types de trous. - Soient $p_{i}(i=1,2)$ les concentrations de trous $i(1,2)$ de masses effectives $m_{i}(i=1,2)$ :

1. LA mobilité de conductivité $\mu_{\mathrm{c}}$ est telle que :

$$
\mu_{\mathrm{c}}\left(p_{1}+p_{2}\right)=\mu_{1} p_{1}+\mu_{2} p_{2} .
$$

Or, $\mu_{i}$ est proportionnelle à $m_{i}^{-1}$ d'après (8) et $\dot{p}_{i}$ est proportionnelle à $\beta_{i}$, donc à $m_{i}^{3 / 2}$ d'après $(3)$.

Soient $m_{1}, p_{1}$ les caractéristiques des trous lourds :

Posant :

$$
\frac{\mu_{\mathrm{c}}}{\mu_{1}}=\frac{1+\frac{\mu_{2}}{\mu_{1}} \cdot \frac{p_{2}}{p_{1}}}{1+\frac{p_{2}}{p_{1}}} .
$$

$$
m=\frac{m_{1}^{*}}{m_{2}^{*}}
$$

$$
\frac{\mu_{\mathrm{c}}}{\mu_{1}}=\frac{1+m^{-1 / 2}}{1+m^{-3 / 2}} .
$$

2. Mobilité de HALl. - On ajoute les contributions des trous :

$$
\begin{aligned}
& J_{1} / E_{1}=q \hbar\left[\langle\tau\rangle \sum_{i} \frac{\beta_{i}}{m_{i}}-\left\langle\tau^{3}\right\rangle \sum_{i} \frac{\beta_{i} \omega_{\mathrm{c} i}^{2}}{m_{i}}\right] \\
& J_{2} / E_{1}=q \hbar\left\langle\tau^{2}\right\rangle \sum_{i} \frac{\beta_{i} \omega_{\mathrm{c} i}}{m_{i}}
\end{aligned}
$$

et négligeant encore les termes en $\mathrm{H}^{2}$ :

$$
\begin{aligned}
\frac{\mu_{\mathrm{H}} H}{c} & =\frac{\left\langle\tau^{2}\right\rangle}{\langle\tau\rangle} \cdot \frac{\sum_{i} \beta_{i} \omega_{\mathrm{c} i} / m_{i}}{\sum_{i} \beta_{i} / m_{i}} \\
\mu_{\mathrm{H}} & =\frac{q}{m_{1}^{*}} \cdot \frac{\left\langle\tau^{2}\right\rangle}{\langle\tau\rangle} \cdot \frac{1+\frac{\beta_{2}}{\beta_{1}} \cdot \frac{\omega_{\mathrm{c} 2}}{\omega_{\mathrm{c} 1}} \cdot \frac{m_{1}^{*}}{m_{2}^{*}}}{1+\frac{\beta_{2}}{\beta_{1}} \cdot \frac{m_{1}^{*}}{m_{2}^{*}}}
\end{aligned}
$$

$\omega_{\mathrm{c} i}$ est proportionnelle à $m_{i}^{-1} \mathrm{~d}$ 'après (4).

Il vient alors :

$$
\mu_{\mathrm{H}}=\frac{q}{m_{1}^{*}} \cdot \frac{\left\langle\tau^{2}\right\rangle}{\langle\tau\rangle} \cdot \frac{1+m^{1 / 2}}{1+m^{-1 / 2}}
$$

et :

$$
\begin{gathered}
\mu_{1}=\frac{q}{m_{1}^{*}} \frac{\left\langle\tau^{2}\right\rangle}{\left\langle\tau^{0}\right\rangle} \\
\frac{\mu_{\mathrm{H}}}{\mu_{1}}=\frac{\left\langle\tau^{2}\right\rangle\left\langle\tau^{0}\right\rangle}{\langle\tau\rangle^{2}} \cdot \frac{1+m^{1 / 2}}{1+m^{-1 / 2}} \\
\frac{\mu_{\mathrm{H}}}{\mu_{1}}=f \cdot \frac{1+m^{1 / 2}}{1+m^{-1 / 2}} .
\end{gathered}
$$

3. Mobilité de Corbino. - On écrit :

$$
\frac{J_{1}}{E_{1}}=\sigma(H)
$$

et la variation relative de résistance :

$$
\begin{gathered}
\frac{\Delta \rho}{\rho_{0}}=\frac{\sigma_{0}-\sigma}{\sigma_{0}}=\frac{\left\langle\tau^{3}\right\rangle}{\langle\tau\rangle} \cdot \frac{\frac{\beta_{1} \omega_{\mathrm{c} 1}^{2}}{m_{1}^{*}}+\frac{\beta_{2} \omega_{\mathrm{c} 2}^{2}}{m_{2}^{*}}}{\frac{\beta_{1}}{m_{1}^{*}}+\frac{\beta_{2}}{m_{2}^{*}}} \\
\left(\frac{\mu_{\mathrm{M}} H}{c}\right)^{2}=\frac{\left\langle\tau^{3}\right\rangle}{\langle\tau\rangle} \cdot \omega_{\mathrm{c} 1}^{2} \frac{1+\frac{\beta_{2}}{\beta_{1}} \cdot \frac{\omega_{\mathrm{c} 2}^{2}}{\omega_{\mathrm{c} 1}^{2}} \cdot \frac{m_{1}^{*}}{m_{2}^{*}}}{1+\frac{\beta_{2}}{\beta_{1}} \cdot \frac{m_{1}^{*}}{m_{2}^{*}}} \\
\mu_{\mathrm{M}}^{2}=\frac{q^{2}}{m_{1}^{* 2}} \cdot \frac{\left\langle\tau^{3}\right\rangle}{\langle\tau\rangle} \cdot \frac{1+m^{3 / 2}}{1+m^{-1 / 2}} \\
\left(\frac{\mu_{\mathrm{M}}}{\mu_{1}}\right)^{2}=\frac{\left\langle\tau^{3}\right\rangle\left\langle\tau^{0}\right\rangle^{2}}{\langle\tau\rangle^{3}} \cdot \frac{1+m^{3 / 2}}{1+m^{-1 / 2}} .
\end{gathered}
$$


Or :

$\frac{\left\langle\tau^{3}\right\rangle\left\langle\tau^{0}\right\rangle^{2}}{\langle\tau\rangle^{3}}=\frac{\left\langle\tau^{3}\right\rangle\langle\tau\rangle}{\left\langle\tau^{2}\right\rangle^{2}} \cdot \frac{\left\langle\tau^{2}\right\rangle^{2}\left\langle\tau^{0}\right\rangle^{2}}{\langle\tau\rangle^{4}}=\theta f^{2}$.

De sorte que :

$$
\frac{\mu_{\mathrm{M}}}{\mu_{1}}=f \sqrt{\theta} \sqrt{\frac{1+m^{3 / 2}}{1+m^{-1 / 2}}}
$$

Combinant (20) et (22) :

$$
\frac{\mu_{\mathrm{M}}}{\mu_{\mathrm{H}}}=\sqrt{\theta} \frac{\sqrt{\left(1+m^{3 / 2}\right)\left(1+m^{-1 / 2}\right)}}{1+m^{1 / 2}}
$$

et combinant $(18)$ et $(22)$, puis $(18)$ et $(20)$ :

$$
\begin{gathered}
\frac{\mu_{\mathrm{M}}}{\mu_{\mathrm{c}}}=f \sqrt{ } \bar{\theta} \cdot \frac{\left(1+m^{3 / 2}\right)^{1 / 2}\left(1+m^{-3 / 2}\right)}{\left(1+m^{-1 / 2}\right)^{3 / 2}} \\
\quad \frac{\mu_{\mathrm{H}}}{\mu_{\mathrm{c}}}=f \cdot \frac{\left(1+m^{1 / 2}\right)\left(1+m^{-3 / 2}\right)}{\left(1+m^{-1 / 2}\right)^{2}} .
\end{gathered}
$$

\section{BIBLIOGRAPHIE}

[1] Grosvalet, JUND, POIRIER et MOTSCH, Surface Science, 1966, 5, 49.

[2] VAN DER PAUw, Philips Research Rep., 1958, 13.

Г२า BEER, Solid State Physics, Suppl. no 4 , Academic Press, 1963.
[4] Jund (C.), C. R. trimestriel de Marché d'Études, 1-10-1968.

[5] Verré (C.), Thèse de $3^{\text {e }}$ cycle, 9 juin 1963 , soutenue auprès de 1 'Université de Paris.

[6] Mercier (J.), Journées de l'Électronique de Toulouse, 3-7 mars 1969, Colloque Microélectronique. 